\title{
Temporal coherence characteristics of a superluminescent diode system with an optical feedback mechanism
}

Fang-Wen Sheu, Pei-Ling Luo

Fang-Wen Sheu, Pei-Ling Luo, "Temporal coherence characteristics of a superluminescent diode system with an optical feedback mechanism," Proc. SPIE 9665, Tenth International Topical Meeting on Education and Training in Optics and Photonics, $96650 Z$ (3 June 2007); doi: 10.1117/12.2207345

SDIE Event: Tenth International Topical Meeting on Education and Training in Optics and Photonics, 2007, Ottawa, Ontario, Canada 


\title{
Temporal coherence characteristics of a superluminescent diode system with an optical feedback mechanism
}

\author{
Fang-Wen Sheu and Pei-Ling Luo \\ Department of Applied Physics, National Chiayi University, Chiayi 60004, Taiwan \\ Tel: +886-5-2717993; Fax: +886-5-2717909; E-mail: fwsheu@ mail.ncyu.edu.tw
}

\begin{abstract}
We explore the temporal coherence characteristics of the output light of a SLD system with different optical feedback ratios by a Michelson interferometer, and we also observe the long-scan-range interference patterns with the one by one wave packets due to the Fabry-Perot modulation of the SLD device. We can obtain the effective cavity length of the SLD active layer and get more information of the temporal coherence length or spectral width from the long-scan-range interference patterns. This tunable light source system can provide more insights into the optical coherence or lasing phenomena often discussed in the optics course.

(C) 2007 Optical Society of America

OCIS codes: (140.2020) Diode lasers; (030.1640) Coherence
\end{abstract}

\section{Introduction}

Broadband light sources such as light-emitting diodes (LEDs) and superluminescent diodes (SLDs) have been widely used for the optical measurement [1], and especially the SLD broadband source has been playing an important role in the optical coherence tomography system [2,3]. High output power and large optical bandwidth are key features for the SLD, and the extremely high optical gain in SLD active region may result in very high optical power sensitivity to external optical feedback [4]. Thus, once stimulated emission due to optical feedback occurs, the output light intensity could be increased to achieve optical amplification, and the evident variation of the spectrum shape and temporal coherence length could also be observed.

In this study, we have constructed an experimental system using a non-fiber-coupled SLD device with optical feedback as the light source to observe the output optical spectra by an optical spectrum analyzer, and to investigate the temporal coherence characteristics of the SLD output light by a Michelson interferometer with the short-scan-range and long-scan-range interference patterns.

\section{Measurement of the output optical spectra}

\subsection{Experimental setup}

We use a SLD broadband light-emitting device (HAMAMATSU 8414-04) [5] as the light source of the experimental system subjected to an optical feedback mechanism, as described schematically in Fig. 1. The output light of the SLD device is collimated by a focusing lens, and then is divided into the reflection arm (70\%) and the output arm (30\%) using a cubic beam splitter (BS). A mirror M is used to reflect the light in the reflection arm back into the SLD device, producing external optical feedback to enhance the stimulated emission light. We can control the optical intensity of feedback by placing a neutral density filter (NDF) between the beam splitter BS and the mirror $\mathrm{M}$. The feedback ratio is equal to $\left(\mathrm{P}_{\mathrm{r}} / \mathrm{P}_{\mathrm{i}}\right)^{2}$, where the $\mathrm{P}_{\mathrm{i}}$ is the initial optical power of the output light emitted directly from the SLD device and the $\mathrm{P}_{\mathrm{r}}$ is the optical power of the light passing through the NDF in the reflection arm. We measure the output spectra of the SLD system at various optical feedback ratios, and then calculate the spectral widths.

Tenth International Topical Meeting on Education and Training in Optics and Photonics, edited by Marc Nantel, Proc. of SPIE Vol. 9665, 96650Z · ( 2007 SPIE, OSA, IEEE, ICO doi: $10.1117 / 12.2207345$ 


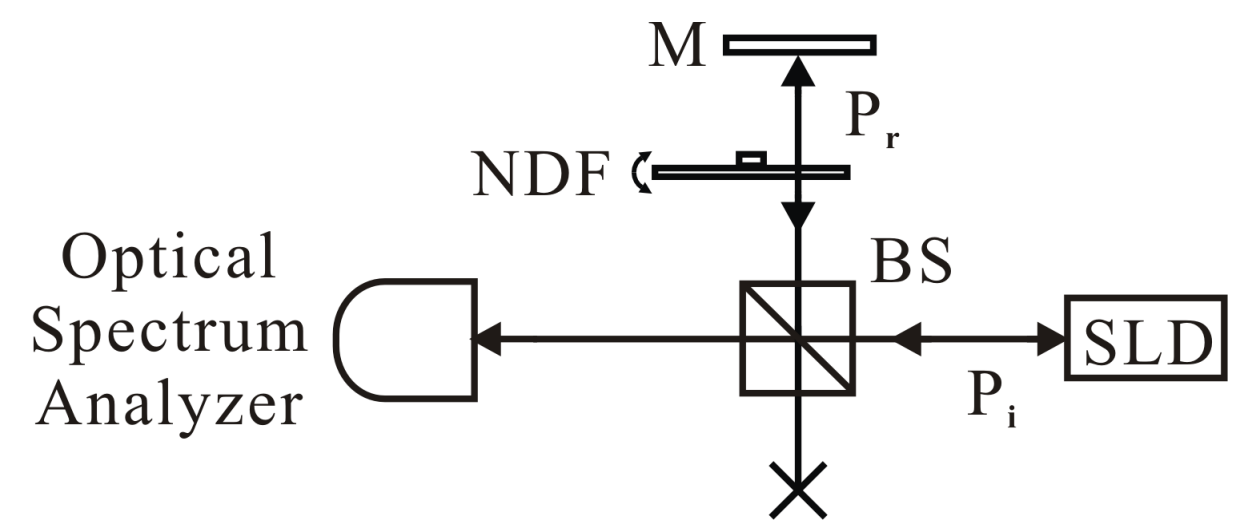

Fig. 1. Experimental setup of the SLD system subjected to a tunable optical feedback mechanism. BS, beam splitter. NDF, neutral density filter. M, mirror.

\subsection{The output spectrum characteristics}

We measure the SLD output spectra by an optical spectrum analyzer with a resolution limit of $0.01 \mathrm{~nm}$. Fig. 2(a) shows the measured spectrum of the spontaneous emission light from a SLD system without optical feedback, and we get that the center wavelength is at $836 \mathrm{~nm}$ and the spectral width is $21.75 \mathrm{~nm}$. The spectrum with the center wavelength at about $838 \mathrm{~nm}$ of the stimulated emission light from a SLD system with optical feedback is shown in Fig. 2(b). The mode spacing of the stimulated emission light is measured to be about $0.343 \mathrm{~nm}$, referring to the internal-cavity longitudinal modes of the SLD device. These internal-cavity resonant modes tell us that the laser oscillation in the SLD system with optical feedback is indeed real. Accordingly, the effective cavity length of the SLD active layer is calculated to be $1.038 \mathrm{~mm}$.

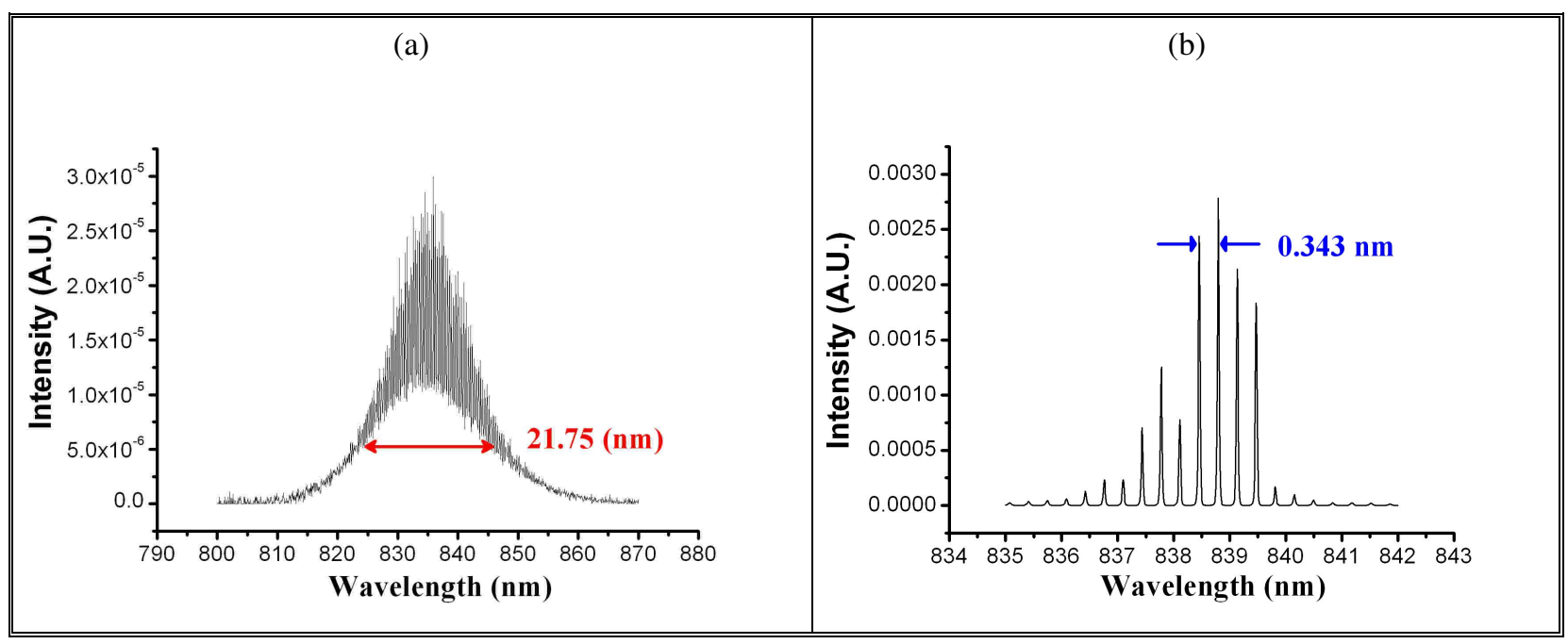

Fig. 2. (a) The spectrum of the spontaneous emission light from a SLD system without optical feedback. (b) The spectrum of the stimulated emission light from a lasing SLD system with optical feedback.

Furthermore, we plot the spectral width (FWHM) of the SLD output spectrum versus different feedback ratios in Fig. 3. As the feedback ratio is raised the spectral width of the SLD output light becomes narrower, because the stimulated emission light due to optical feedback will compete for the gain. However, having excessive optical feedback can cause the spectral width to become broader. From this relationship, we can know that the spectral width of the SLD output spectrum can be tuned via adjusting the feedback ratio. As a result, we deduce that the temporal coherence characteristics of the output light from a SLD system with optical feedback should be also tunable, which will be explored in the next section. 


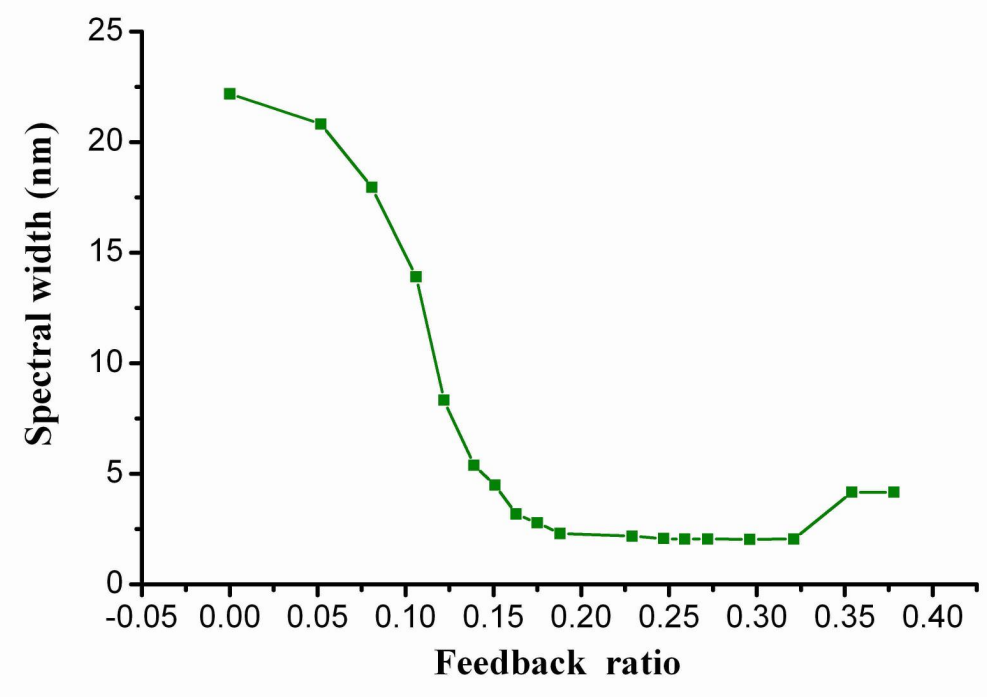

Fig. 3. The spectral width (FWHM) versus the feedback ratio curve.

\section{Investigation of the temporal coherence characteristics by a Michelson interferometer}

\subsection{Experimental setups}

We construct a SLD system subjected to an optical feedback mechanism, and then utilize a Michelson interferometer in the output arm to observe interference patterns and measure the temporal coherence length by moving one of the mirrors, as shown in Fig. 4. We monitor the interference signals by an oscilloscope for two cases, namely one is with short-scan-range and another is with long-scan-range. Besides, we also explore the temporal coherence characteristics of the output light of the SLD system at various optical feedback ratios.

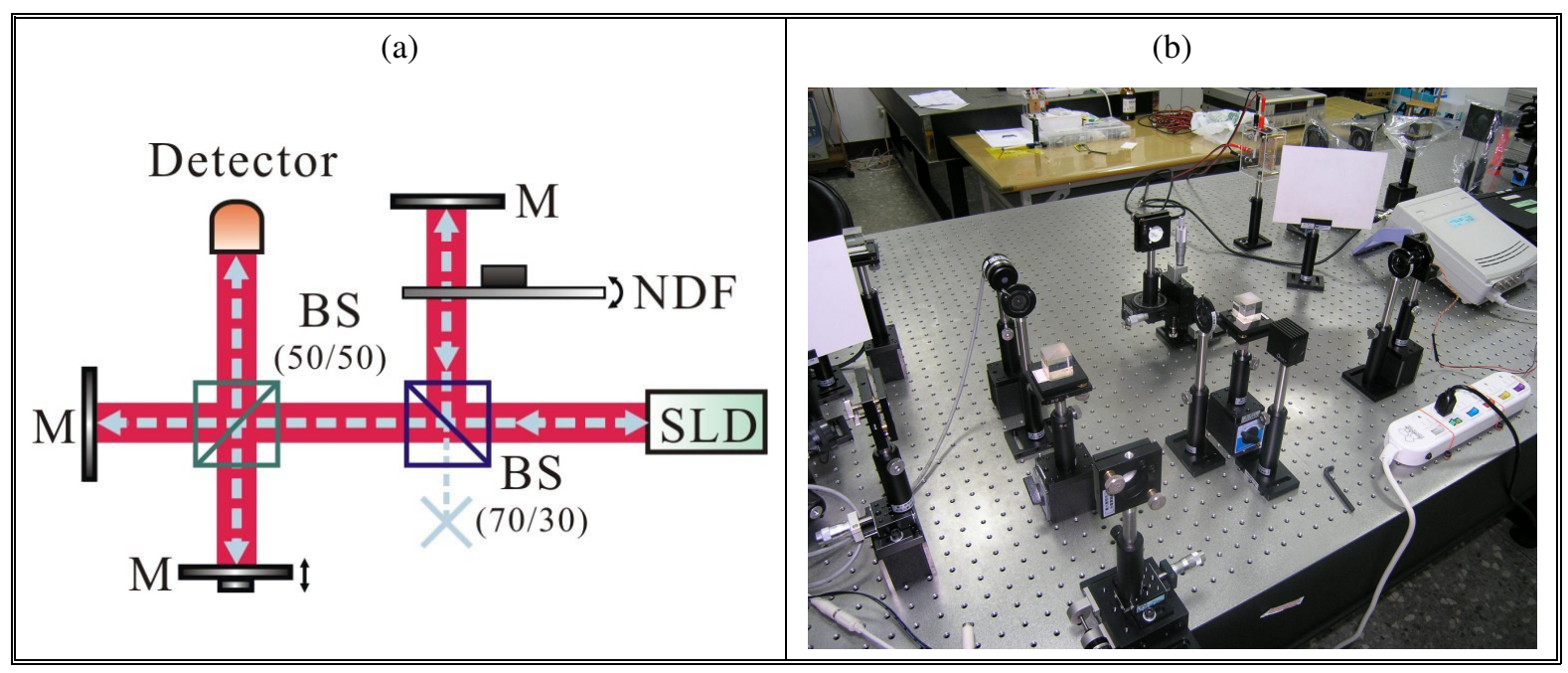

Fig. 4. (a) The schematic diagram of the experimental setup for observing the degree of temporal coherence. BS (70/30), beam splitter with the ratio of separating light of reflection and transmission at 70:30. BS (50/50), beam splitter with the ratio of separating light at 50:50. NDF, neutral density filter. M, mirror. (b) The photograph of the experimental setup. 


\subsection{The short-scan-range interference results}

We first observe the short-scan-range interference patterns of the spontaneous emission light from a SLD system without optical feedback, and those of the stimulated emission light from a SLD system with maximum optical feedback. Figure 5 shows the experimental setups and measurement results. As shown in Fig. 5(a), the measured coherence length of the spontaneous emission light of SLD equals $14.285 \mu \mathrm{m}$ which is almost identical to the theoretical value $\mathrm{L}_{\mathrm{c}}=0.44 \times \lambda_{0}{ }^{2}{ }_{I \Delta} \lambda=14.14 \mu \mathrm{m}$, where $\lambda_{0}=836 \mathrm{~nm}$ and ${ }_{\Delta} \lambda=21.75 \mathrm{~nm}$. The error is about $1.025 \%$, so the measured value by interference is very precise. Another case with maximum optical feedback is shown in Fig. 5(b) with much longer coherence length, and the measured scan distance between two adjacent peaks of interference signal is $425.6 \mathrm{~nm}$, which is just about one half of the center lasing wavelength $(\lambda=838$ $\mathrm{nm}$ ), fulfilling the requirement of a Michelson interferometer. The error is about $1.575 \%$, so the measured value of the lasing wavelength by interference is almost identical with the theoretical value.

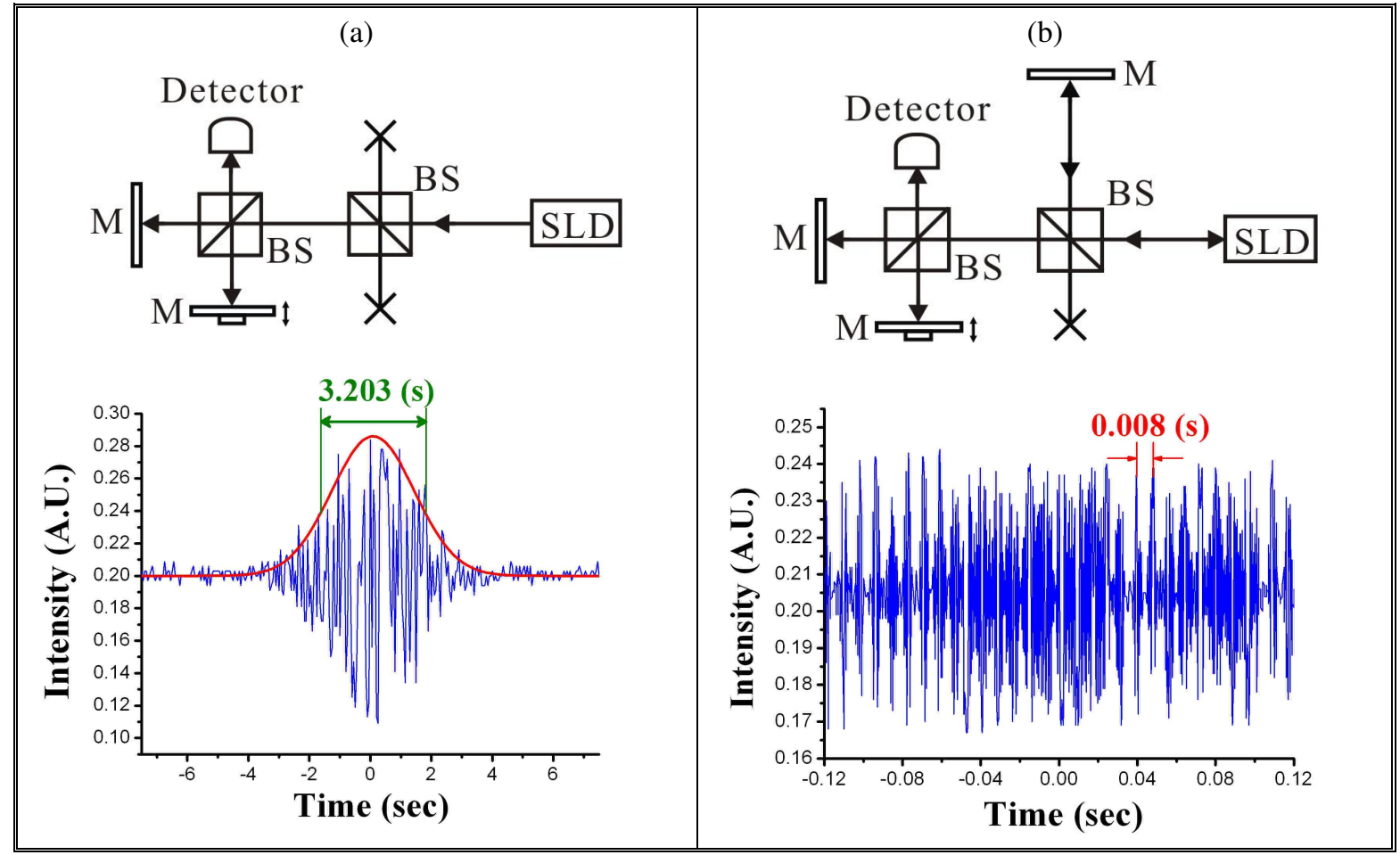

Fig. 5. Experimental setups (top) and measured interference patterns (bottom) for (a) the case without optical feedback at a constant scan speed of $4.46 \mu \mathrm{m} / \mathrm{s}$, and for (b) another case with maximum optical feedback at a constant scan speed of $53.2 \mu \mathrm{m} / \mathrm{s}$.

\subsection{The long-scan-range interference results}

We next observe the long-scan-range interference patterns with one by one wave packets of the SLD system at various optical feedback ratios. The one by one wave packets of interference patterns are caused by the Fabry-Perot modulation of the SLD device, and the schematic diagram of the optical paths due to multiple reflections in the SLD active layer is shown in Fig. 6(a). We assume that the output light of the SLD system can be imagined effectively as multiple wave packets by analogy. For different degrees of temporal coherence of output light, the widths of the effective wave packet and the corresponding interference patterns are different. In Fig. 6(b), there is the long-scan-range interference pattern of the spontaneous emission light of SLD. From the wave packet model, the value of wave packet separation is $1.036 \mathrm{~mm}$, which equals the effective cavity length of the SLD active layer, $\mathrm{nL}_{\mathrm{a}}$, where $\mathrm{L}_{\mathrm{a}}$ is the length of the SLD active layer and $\mathrm{n}$ is effective refractive index for 
optical mode. The long-scan-range interference pattern of the stable laser output of a SLD system with an optical feedback ratio at 0.25 has a larger interference wave packet width due to a longer temporal coherence length, as shown in Fig. 6(c). The interference pattern for the case with an optical feedback ratio at 0.37 has a separation of wave packets similar to the case without feedback, but has a broader expansion of multiple wave packets due to the higher optical power output when the SLD system is in lasing resonance, as shown in Fig. 6(d). Hence the temporal coherence length (or the interference wave packet width) of the output light of the SLD system can be tuned by varying the feedback ratio. The relationship of the temporal coherence length of the long-scan-range interference pattern versus different feedback ratios is found to be similar to that of the spectral width of the SLD output spectrum versus different feedback ratios.

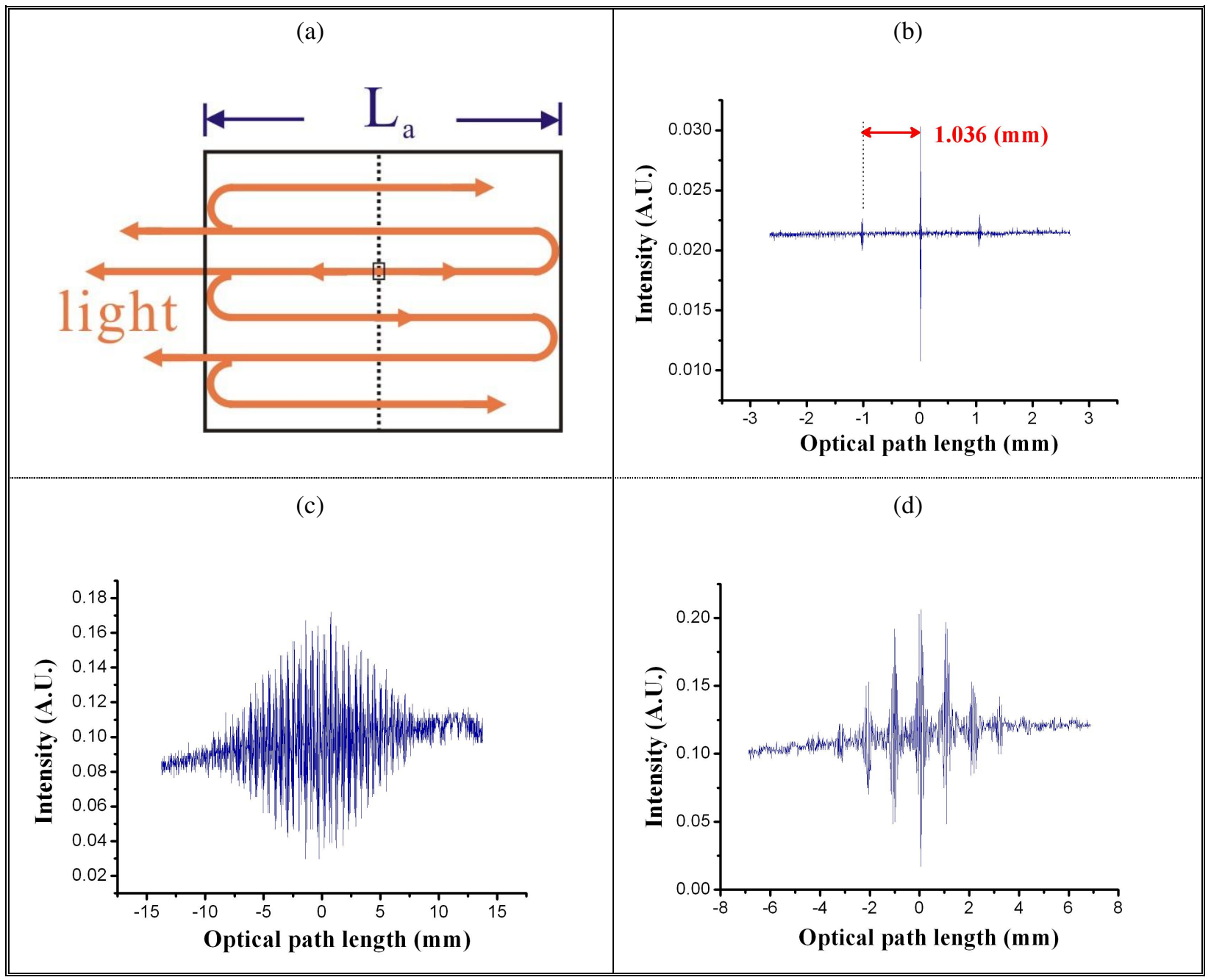

Fig. 6. (a) The schematic diagram of the optical paths due to multiple reflections in the SLD active layer. The long-scan-range interference patterns of the SLD system with different optical feedback ratios: (b) feedback ratio at 0 , (c) feedback ratio at 0.25 , and (d) feedback ratio at 0.37 . 


\section{Conclusion}

The evident variation of the temporal coherence characteristics between the spontaneous emission and the stimulated emission output light of the SLD system could be observed by a Michelson interferometer. By adjusting the feedback ratio, we could obtain different types of long-scan-range interference patterns with variable multiple interference wave packets due to the Fabry-Perot modulation of SLD device. From these interference patterns, we can estimate the effective cavity length of the SLD active layer. This tunable light source system can provide more insights into the optical coherence or lasing phenomena often discussed in the optics course.

\section{Acknowledgements}

We acknowledge the financial support from the National Science Council, Taiwan, through project NSC-95-2112-M-415-004.

\section{References}

[1] M. Fukuda, "Optical semiconductor devices", (John Wiley \& Sons, 1999).

[2] D. Huang, E.A. Swanson, C.P. Lin, J.S. Schuman, W.G. Stinson, W. Chang, M.R. Hee, T. Flotte, K. Gregory, C.A. Puliafito, and J.G. Fujimoto, "Optical coherence tomography", Science 254, 1178 (1991).

[3] U. Morgner, W. Drexler, F.X. Kärtner, X.D. Li, C. Pitris, E.P. Ippen, and J.G. Fujimoto, "Spectroscopic optical coherence tomography", Opt. Lett. 25, 111 (2000)

[4] V. Shidlovski, "Superluminescent Diodes. Short overview of device operation principles and performance parameters", (SuperlumDiodes Ltd., 2004). http://www.superlumdiodes. com/

[5] http://www.hamamatsu.com/ 